\title{
ARTÍ́CULOS
}

Sometido 06-06-2020. Aprobado 22-06-2021

Evaluado por el sistema double blind review. Editor científico invitado: Ricardo Teixeira Veiga

Versión original | DOI: http://dx.doi.org/10.1590/S0034-759020220301

\section{COLABORACIÓN EXTERNA DE LA INNOVACIÓN ABIERTA Y DESEMPEÑO FINANCIERO DE LAS PYMES EN TAMAULIPAS, MÉXICO}

\section{Colaboração externa da inovação aberta e desempenho financeiro das PMEs no Tamaulipas, México External collaboration of open innovation and financial performance of SMES
in Tamaulipas, Mexico}

Erick Leobardo Alvarez-Aros ${ }^{1}$ | erickleobardo.alvarez@upaep.mx | ORCID: 0000-0002-1934-5442 César Augusto Bernal-Torres² | cesar.bernal@unisabana.edu.co | ORCID: 0000-0002-7410-1109 Yesenia Sánchez Tovar ${ }^{3}$ | yesanchez@docentes.uat.edu.mx | ORCID: 0000-0002-4299-191X

${ }^{1}$ Universidad Popular Autónoma del Estado de Puebla, Programs in Strategic Planning and Technology Management, Puebla, Puebla, Mexico ${ }^{2}$ Universidad de La Sabana, International School of Economic and Administrative Sciences, Chía, Cundinamarca, Colombia

${ }^{3}$ Universidad Autónoma de Tamaulipas, Division of Graduate Studies and Research, Victoria City, Tamaulipas, Mexico

\section{RESUMEN}

La innovación abierta es un paradigma colaborativo que mejora el desempeño empresarial. Sin embargo, la mayoría de las investigaciones abordan empresas grandes y multinacionales de economías desarrolladas, por lo que este trabajo analizó la relación de la colaboración externa de la innovación abierta con el desempeño financiero en pequeñas y medianas empresas en una economía emergente. Para ello, se aplicó un cuestionario a propietarios y directivos de 145 empresas localizadas en Tamaulipas, México. Los resultados procesados mediante ecuaciones estructurales en SPSS y AMOS indican ausencia de relación positiva y significativa entre la colaboración vertical de la innovación abierta (clientes, usuarios y proveedores) con el desempeño financiero. En contraste, se comprobó una relación positiva y significativa entre la colaboración horizontal de la innovación abierta (instituciones de educación, gobierno y competidores) con el desempeño financiero, con elementos como el codesarrollo de productos, entradas de conocimiento e ideas, adquisición de maquinaria, equipo, software e insumos.

Palabras Clave: Innovación abierta, colaboración horizontal, colaboración vertical, desempeño financiero, economía emergente.

\section{RESUMO}

A inovação aberta é um paradigma colaborativo que melhora o desempenho dos negócios. No entanto, a maioria das investigações aborda empresas grandes e multinacionais com economias desenvolvidas. Este trabalho analisou a relação da colaboração externa da inovação aberta com o desempenho financeiro em pequenas e médias empresas em uma economia emergente. Para isso, foi aplicado um questionário a proprietários e gerentes de 145 empresas localizadas em Tamaulipas, no México. Os resultados processados por meio de equações estruturais no SPSS e AMOS indicam a ausência de uma relação positiva e significativa entre a colaboração vertical da inovação aberta (clientes, usuários e fornecedores) e o desempenho financeiro. Em contraste, uma relação positiva e significativa foi encontrada entre a colaboração horizontal da inovação aberta (instituições educacionais, governo $e$ concorrentes) com o desempenho financeiro, com elementos como o codesenvolvimento de produtos, insumos de conhecimento e ideias, aquisição de máquinas, equipamentos, softwares e suprimentos. Palavras-Chave: Inovação aberta, colaboração horizontal, colaboração vertical, desempenho financeiro, economia emergente.

\section{ABSTRACT}

Open innovation is a collaborative paradigm that improves business performance. However, the majority of investigations approach large and multinational companies with developed economies, so this work analyzed the relationship of external collaboration of open innovation with financial performance in small and medium-sized companies in an emerging economy. For this, a questionnaire was applied to owners and managers of 145 companies located in Tamaulipas, Mexico. The results processed through structural equations in SPSS and AMOS indicate the absence of a positive and significant relationship between the vertical collaboration of open innovation (customers, users and suppliers) and financial performance. In contrast, a positive and significant relationship was found between the horizontal collaboration of open innovation (educational institutions, government and competitors) with financial performance, with elements such as co-development of products, inputs of knowledge and ideas, acquisition of machinery, equipment, software and supplies.

Keywords: Open innovation, horizontal collaboration, vertical collaboration, financial performance, emerging economy. 


\section{INTRODUCCIÓN}

Actualmente, la innovación tradicional o cerrada es insuficiente, sin embargo, surge la innovación abierta como alternativa de investigación y desarrollo (I+D) para obtener conocimientos y recursos que mejoran el desempeño financiero por colaboración externa, obteniendo así ventajas competitivas (Alvarez-Aros \& Álvarez-Herrera, 2018; Chesbrough, 2003; Chesbrough, Vanhaverbeke, \& West, 2006; Enkel, Gassmann, \& Chesbrough, 2009; García-Vidales, Maldonado-Guzmán, \& PinzónCastro, 2019).

Esto manifiesta una innovación abierta complementaria a la I+D interna, caracterizada por experiencia y capacidad laboral. En cambio, la innovación abierta colabora externamente, fuera de los limites organizacionales, obteniendo ideas y conocimiento para enriquecer los procesos innovadores y el desempeño financiero (Chesbrough, 2006; Greco, Grimaldi, \& Cricelli, 2015; Schroll \& Mild, 2011).

No obstante, aunque la innovación abierta es más aceptada en la academia, el mundo empresarial sigue explorando el enfoque colaborativo, pues este no ha logrado una contundente contribución al desempeño financiero de cualquier índole de empresas y sectores (Chesbrough, 2003, 2015; Dahlander \& Gann, 2010).

La mayoría de los estudios sobre innovación abierta se concentran en empresas grandes y multinacionales del sector de tecnología y software. Por consiguiente, el estudio en pequeñas y medianas empresas (pymes) y otros sectores no ha demostrado ampliamente los beneficios generados (Calderón, 2010; Krause \& Schuttle, 2015; Raposo, Ferreira, \& Fernandes, 2014; Stanisławski, 2020; Yoon, Shin, \& Lee, 2016).

Como ejemplo, las pymes colombianas y venezolanas resaltan la importancia de la colaboración externa, pero realizan una innovación tradicionalista con aperturas menores hacia proveedores y clientes. Es decir, las empresas trabajan la I+D interna, en vez de colaborar externamente y buscar otras fuentes de conocimiento y recursos (Bernal-Torres \& Frost-González, 2015; Rodríguez, Terán, \& Bucci, 2011).

En una economía emergente como México, la innovación y sus prácticas representan una pieza clave para el desempeño y el sostenimiento empresarial (Armenteros, Elizondo, Medina, Ballesteros, \& Molina, 2012; Maldonado-Guzmán, Madrid-Guijarro, Martínez-Serna, \& AguileraEnríquez, 2009; Souza, Torres, \& Miyake, 2018), sin embargo, en cuanto a innovación abierta se refiere, existen pocas investigaciones que sustentan las colaboraciones externas con el desempeño organizacional.

En tamaño empresarial, las pymes contribuyen mayoritariamente en la economía local y la generación de empleos, al tener más unidades económicas pequeñas donde se enfrentan a retos mayores a las multinacionales (Andersen, 1999; Armenteros et al., 2012; Fred, 2017; MarínIdárraga \& Cuartas-Marín, 2019; Sánchez, Zerón \& Mendoza, 2015; Ugarte-Cataldo, 2013; Vieira, 2014).

En México, el Instituto Nacional de Estadística y Geografía (Inegi) en 2016, registraba 4.2 millones de unidades económicas, el $99.8 \%$ eran pymes y generaban el $52 \%$ del producto interno bruto (PIB) y el 78\% de la tasa de empleo (Inegi, 2016). Según datos anuales de 1989, 
1994, 1999, 2004, 2009 y 2014, el promedio de supervivencia de nuevos negocios en México es de 7.8 años, pero en Tamaulipas es de 6.4 años, ubicándose entre los últimos estados.

Tamaulipas aporta el 3\% del PIB, según el Directorio Estadístico Nacional de Unidades Económicas (Denue) y es una región relevante por su ubicación geográfica respecto a los Estados Unidos de América (Denue, \& Inegi, 2019). De esta manera, destaca la importancia de las pymes en la economía y los empleos, y se debe mejorar su supervivencia y desempeño.

Por consiguiente, esta investigación tuvo como propósito analizar la relación de la colaboración externa de la innovación abierta con el desempeño financiero pyme en la frontera norte de México, aportando novedad y originalidad. Para ello, metodológicamente se aplicó un cuestionario y una modelación de ecuaciones estructurales (SEM, por sus siglas en inglés) con el software SPSS y AMOS. Esta investigación presenta la fundamentación teórica, el método, los resultados, y las conclusiones.

\section{COLABORACIÓN EXTERNA Y DESEMPEÑO FINANCIERO}

\section{Colaboración externa}

Desde la innovación abierta, las colaboraciones externas representan una estrategia para complementar las actividades de I+D, y facilitan la exploración tecnológica, la generación de ideas y conocimientos, y la adquisición de recursos que mejoran el desempeño organizacional (Faems, Visser, Andries, \& Looy, 2010; Greco et al., 2015; Mazzola, Bruccoleri, \& Perrone, 2012; Rogo, Cricelli, \& Grimaldi, 2014; Sisodiya, Johnson, \& Grégoire, 2013).

La colaboración se dimensiona en colaboraciones externas verticales y horizontales, con la finalidad de obtener ideas, conocimiento, tecnología y oportunidades (Parida, Westerberg, \& Frishammar, 2012; Vrande, Jong, Vanhaverbeke, \& Rochemont, 2009; Wang, Chang, \& Shen, 2015). La colaboración con usuarios, clientes y proveedores se denomina colaboración externa vertical (Bueno \& Balestrin, 2012; Chatenier, Verstegen, Biemans, Mulder, \& Omta, 2010; Chesbrough et al., 2006; Gassmann, Sandmeier, \& Wecht, 2006; Henkel, 2006). La colaboración con instituciones educativas, gobierno y competidores se denomina colaboración externa horizontal (Cancino \& Cárdenas, 2018; Lee, Park, Yoon, \& Park, 2010).

Las colaboraciones externas verticales cooperan con agentes exógenos, como usuarios, clientes y proveedores, y aprovechan recursos como el conocimiento. De esta forma, influye en la empresa en la medida en que ésta se pueda conectar con los agentes externos en el proceso de innovación, e intercambiar experiencias y soluciones, por transferencia de conocimiento (Gassmann et al., 2006; Hippel, 2005; Schweisfurth \& Raasch, 2015).

Además, los usuarios reducen costos en generación de ideas innovadoras, beneficiándose mutuamente. Las empresas estimulan a usuarios para codesarrollar productos a través de estrategias como el código abierto, donde la tecnología queda a disposición del público para colaborar sin garantías (Bueno \& Balestrin, 2012; Henkel, 2006; Hienerth, 2006). 
Por otro lado, se accede a recursos por interacción con clientes que proporcionan conocimiento externo más detallado de las necesidades empresariales, como los procesos de innovación donde intervienen varias áreas empresariales que capturan eficientemente ideas para satisfacer las necesidades del cliente y crear nuevos o mejorados productos y servicios (Hippel, 2005; Khanagha, Volberda, \& Oshri, 2016; Schweisfurth \& Raasch, 2015).

Dentro de las colaboraciones externas verticales se encuentran los proveedores, que proporcionan grandes beneficios en los procesos de innovación, ya sea, debido a falta de insumos externos con que las empresas no cuentan en sus procesos de aprendizaje, o también trabajando en soluciones conjuntas de materias primas, tiempos de entrega, garantías (Chatenier et al., 2010; Romijn \& Albaladejo, 2002).

Por su parte, en las colaboraciones externas horizontales se realizan conjuntamente esfuerzos de innovación para crear y mantener un desempeño superior, además, se adquiere conocimiento externo que brinda diversidad de ideas y capacidades tecnológicas (Cancino \& Cárdenas, 2018; Hagedoorn, Roijakkers, \& Kranenburg, 2006; Lee et al., 2010; Tether, 2003).

En las colaboraciones externas horizontales la investigación académica ha sido fundamental para acelerar los resultados de innovación y desempeño empresarial (George, Zahra, \& Wood, 2002; Vrande et al., 2009). Las empresas adquieren conocimiento de universidades, tomando en cuenta el ciclo de vida tecnológico, para tomar decisiones respecto al número de personas involucradas, los recursos asignados, el posicionamiento dentro o fuera de los límites de la empresa, el grado de especialización y el grado de formalización entre otros (Buganza \& Verganti, 2009).

Las colaboraciones externas horizontales con gobierno promueven políticas, apoyos económicos, asesoría empresarial para mejora de productos y servicios, nuevos mercados, empresas conjuntas, asociaciones, alianzas y consorcios de I+D para gestionar el conocimiento organizacional, sin descartar las redes informales para crear y gestionar conocimiento (Feller, Finnegan, Hayes, \& O’Reilly, 2009; Lee, Hwang, \& Choi, 2012; Pipkin, 2018).

La colaboración externa horizontal con competidores es cada vez más usada porque se comparten tecnologías, clientes y mercados, no sólo se adquiere tecnología y conocimientos, sino que se accede a otras fuentes de conocimiento globales donde la finalidad es el desarrollo integral de tecnologías, la creación de nuevos mercados, el descubrimiento de nuevas oportunidades de negocio, el aumento de las ganancias en la búsqueda de innovación y un mejor rendimiento organizacional (Lee et al., 2010; Parida et al., 2012).

Por lo tanto, las empresas colaborativas desarrollan características específicas que aumentan su eficiencia a través de la adquisición tecnológica y conocimientos complementarios, además, las estrategias de colaboración aumentan el uso del capital humano y los recursos, por lo que es necesario fijar límites que prevengan la escasez de recursos y satisfacer las necesidades contraídas y sus interacciones (Alvarez-Aros \& Bernal-Torres, 2017; Mention \& Asikainen, 2012; Quintana-García \& Benavides-Velasco, 2004).

En síntesis, las colaboraciones con agentes externos horizontales como verticales representan nuevas ventajas y oportunidades en áreas como la innovación abierta, representando una invitación a la apertura organizacional fuera de los límites empresariales, como se observa en la Figura 1: 
Figura 1. Colaboración externa de la innovación abierta

Fura colaboraton externa de la innovaciòn abierta

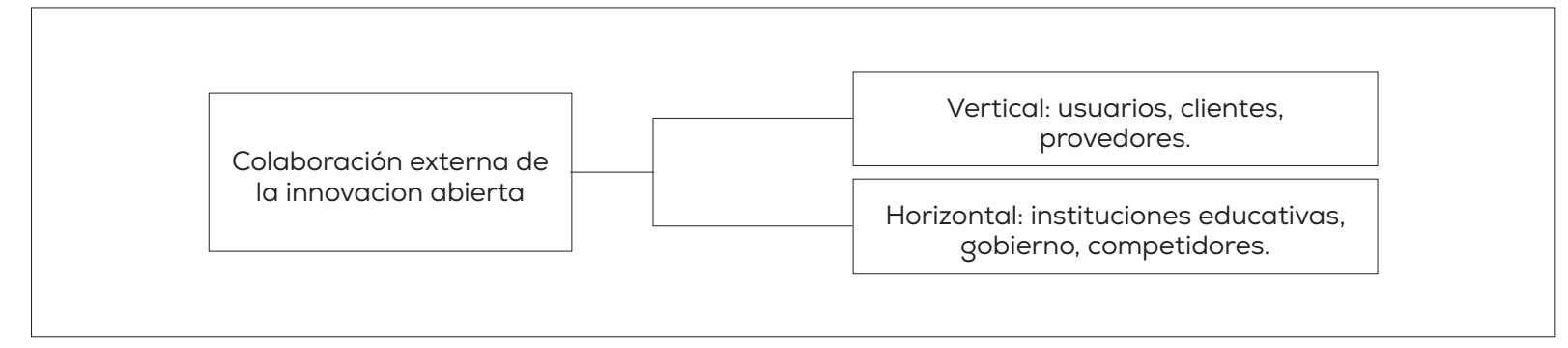

Fuente: Elaboración a partir de Parida et al. (2012); Vrande et al. (2009); Wang et al. (2015).

De la Figura 1, se observa que la colaboración se clasifica según se soporte con cada agente externo, sin embargo, depende de la estrategia de cada organización determinar qué colaboraciones mejoran la innovación y el desempeño financiero, tema abordado en el siguiente apartado.

\section{Desempeño financiero}

Un buen desempeño se sostiene de la viabilidad financiera, la efectividad, la eficiencia y la productividad organizacional. $\mathrm{Al}$ respecto, la medición del desempeño organizacional ha sufrido cambios en el tiempo, sin embargo, la perspectiva financiera sigue vigente en temas como la innovación abierta (Fry, Mention, Temel, \& Torkkeli, 2016; Hung \& Chou, 2013). Como ejemplo, Mazzola, Bruccoleri y Perrone (2016) estudiaron empresas biofarmacéuticas y su mezcla en las prácticas de innovación abierta para extender los procesos de I+D más allá de los límites empresariales y mejorar los resultados financieros.

Otros ejemplos del beneficio de las colaboraciones externas son P\&G, IBM, Intel, Safer, Philips, Unilever y Whirpool, donde se resaltan logros económicos, de adquisición, aplicación y resguardo de ideas, nuevos conocimientos científicos y tecnológicos, la interacción de los canales de comunicación de redes con agentes externos y una flexibilidad organizacional para enfrentar adversidades (Chesbrough, 2015; Muller \& Hutchins, 2012; Ollila \& Yström, 2015).

En este sentido, una mayor flexibilidad empresarial con capacidad de respuesta y de adaptación al entorno otorga mayores rendimientos financieros, tanto a la empresa como a sus colaboradores de la innovación abierta, como lo argumentan estudios empíricos que resaltan el efecto de los derrames de red de conocimientos, pues son recursos clave que mejoran la capacidad relacional con las conexiones externas (Ollila \& Yström, 2015; Sisodiya et al., 2013).

Por lo tanto, a pesar de que el desempeño organizacional ha sido medido diferente en su evolución, la dimensión financiera fue elegida para el estudio porque representa un gran interés para las pymes y la innovación abierta (Akhisar, Tunay, \& Tunay, 2015; Laursen \& Salter, 2006). Al respecto, los indicadores que miden el desempeño financiero se basan en el retorno sobre la inversión (ROI), retorno sobre activos (ROA), crecimiento en ventas, las utilidades, ganancias por acción, valor de mercado y el valor contable (Akhisar et al., 2015; Kalkan, Bozkurt, \& Arman, 2014; Sánchez et al., 2015). 


\section{Colaboración externa y desempeño financiero}

El desempeño financiero y su relación con las colaboraciones externas plantea que una empresa mejora en sus resultados mediante la interacción con agentes externos verticales y horizontales (Hung \& Chiang, 2010; Laursen \& Salter, 2006; Mazzola et al., 2016). De este modo, la colaboración externa vertical con clientes y proveedores es beneficiosa para la innovación de la empresa debido a la combinación de capacidades tecnológicas complementarias y los objetivos comunes con agentes externos (Hwang \& Lee, 2010; Tsai, 2009).

Además, la colaboración externa horizontal con centros de investigación y universidades impacta positivamente el desempeño innovador de productos (Hung \& Chiang, 2010; Tsai, 2009); pues estos agentes cuentan con mecanismos para acceder a nuevos conocimientos. Del lado opuesto, otras investigaciones corroboraron que la adquisición externa de conocimiento tiene un efecto negativo en los resultados organizacionales e innovación (Inauen \& Schenker-Wicki, 2011).

También, la apertura a universidades en los procesos de I+D tiene un impacto positivo en el porcentaje de ventas de productos innovadores, por lo tanto, representa un efecto positivo sobre el desempeño financiero (Inauen \& Schenker-Wicki, 2011). No obstante, investigadores como Belderbos, Faems, Leten y Looy (2010) sugieren la posibilidad de un efecto negativo de estas prácticas en el desempeño financiero, si no se mantienen los limites organizacionales.

De hecho, aunque las actividades colaborativas de $\mathrm{I}+\mathrm{D}$ podrían reducir los riesgos técnicos y los costos asociados a la incertidumbre, la colaboración externa podría introducir riesgos relacionales y el aumento de costos de coordinación (Das \& Teng, 1998). Para mitigar tales riesgos, las empresas requieren de negociaciones contractuales que podrían consumir mucho tiempo para su diseño y aplicación, así como para formular mecanismos de monitoreo generalmente costosos.

Desde lo anterior, existen razones multifacéticas de esta relación negativa, incluyendo la capacidad de absorción inadecuada o insuficiente (Cohen \& Levinthal, 1990; Martínez-Senra, Quintás, \& Caballero, 2014) para absorber conocimiento y tecnologías de otras industrias, o el desvanecimiento de recursos que la adquisición de conocimiento externo crea. Además de impactar el desempeño innovador (Aschhoff \& Schmidt, 2008), algunos trabajos empíricos descritos muestran que la colaboración con agentes externos tiende a ser beneficiosa, no sólo en innovación, sino también en desempeño financiero. Por lo tanto, a raíz del cisma de las consideraciones anteriores, se plantean las siguientes hipótesis:

Hla: La colaboración externa vertical de la innovación abierta influye positiva y significativamente en el desempeño financiero de las pymes.

H1b: La colaboración externa horizontal de la innovación abierta influye positiva y significativamente en el desempeño financiero de las pymes.

Las hipótesis y la representación del modelo se aprecian en la Figura 2. 
Figura 2. Modelo conceptual e hipótesis

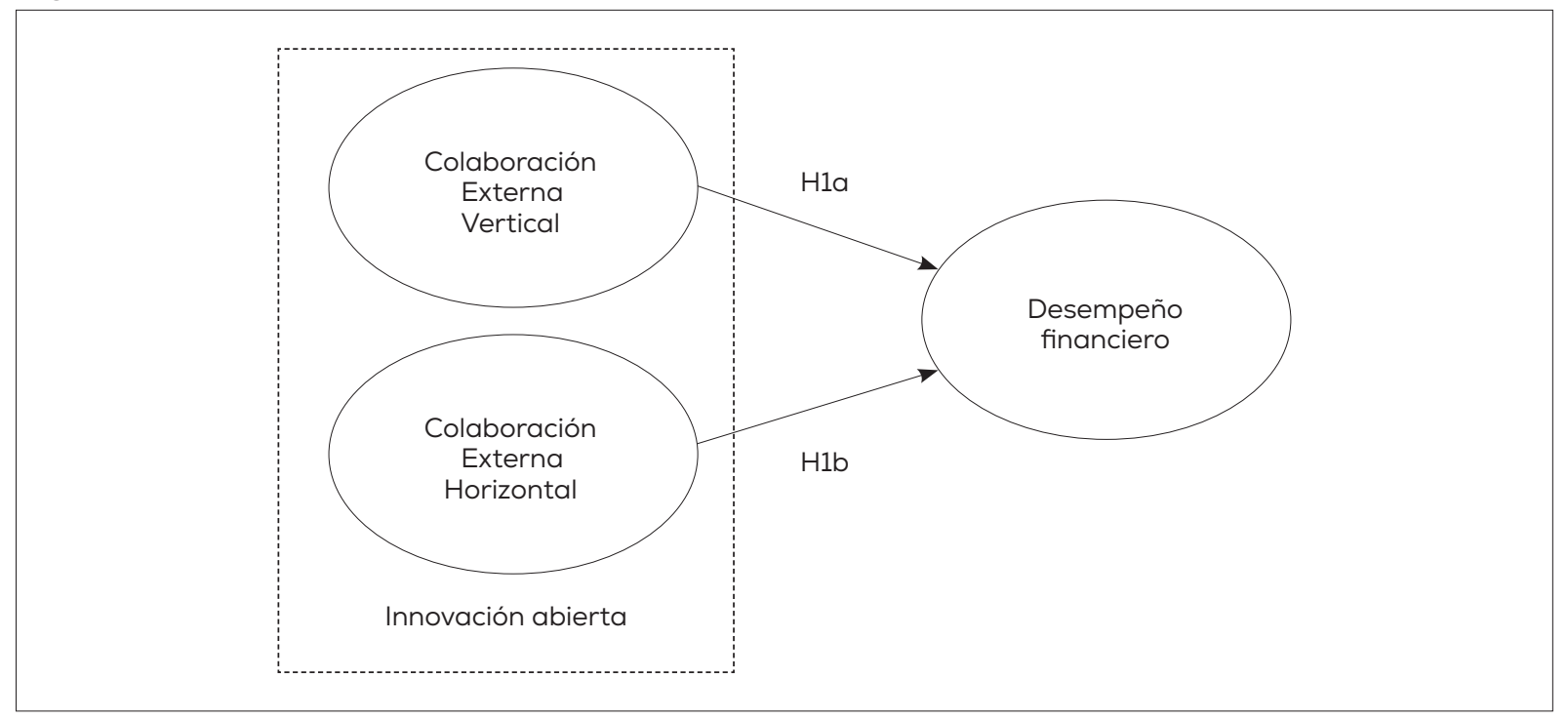

\section{DISEÑO METODOLÓGICO}

\section{Método}

Para la comprobación de las hipótesis planteadas se diseñó una investigación de enfoque cuantitativo, de alcance causal, corte transversal, a través de la recolección de datos con fuentes primarias por medio de un cuestionario. Antes de aplicar el cuestionario definitivo se realizó una prueba piloto con 30 empresas tamaulipecas para identificar posibles problemas de interpretación, garantizar el correcto uso de la escala y lograr la validez del instrumento. La recopilación de datos fue mediante una encuesta en escala Likert de 5 puntos, constituida por 14 ítems relativos a la medición de la colaboración externa tanto vertical como horizontal y siete ítems para medir el desempeño financiero.

El cuestionario se conformó de dos apartados. El primero, recopiló la información general, como el puesto, escolaridad, sexo, sector empresarial y tamaño empresarial. El segundo, recopiló datos de la colaboración externa y el desempeño financiero.

En la colaboración externa vertical, se usaron 7 ítems relacionados con el involucramiento empresarial para investigar, desarrollar o innovar con: clientes actuales; clientes potenciales; usuarios actuales; usuarios potenciales; proveedores actuales; proveedores potenciales; y si el involucramiento se realiza a través de un servicio web o plataformas digitales para facilitar las colaboraciones con los usuarios, los clientes, y los proveedores. El diseño se fundamentó en Afcha (2011), Obea (2009), y Vrande et al. (2009).

En la colaboración externa horizontal, los 7 ítems utilizados se relacionaron con el involucramiento empresarial con: instituciones educativas; gobierno; competidores; trabajo 
conjunto de actividades como el codesarrollo de productos o servicios con instituciones educativas, o gobierno o competidores; uso de redes de intercambio de experiencias con instituciones educativas, o gobierno o competidores; obtención de entradas de conocimiento e ideas con instituciones educativas, o gobierno o competidores; entradas de equipo, maquinaria, software, e insumos con instituciones educativas, o gobierno o competidores. Los ítems se fundamentaron en Afcha (2011), Obea (2009), Vrande et al. (2009) y Wang et al. (2015).

Para los ítems de la colaboración externa vertical y horizontal, las opciones de la escala fueron: uno "nunca”, dos "rara vez", tres “algunas veces”, cuatro "la mayoría de las veces" y cinco "siempre".

Para evaluar el desempeño financiero, los ítems utilizados se relacionaron con la rentabilidad; la cobranza a clientes; el pago a proveedores; la rotación de los inventarios; el retorno sobre los activos; el retorno sobre las ventas; y el retorno sobre la inversión. Para dichos ítems se tomaron en cuenta los trabajos de Martínez, Charterina y Araujo (2010), Park y Lee (2011), Rogo et al. (2014) y Sisodiya et al. (2013). Las opciones de la escala fueron: uno "pésimo”, dos "malo", tres "regular", cuatro "bueno" y cinco "superior".

El cuestionario fue aplicado a una muestra no probabilística a conveniencia (sin inferencia estadística) y fue enviado por correo electrónico a 250 directivos, es decir, dueños, propietarios, gerentes generales y financieros de las pymes del sector industrial, comercial y servicios en Tamaulipas, México. Previo al envío, se contactó a cámaras y asociaciones que proporcionaron los contactos y correos electrónicos. Se recibieron 170 encuestas, pero se descartaron 25 por problemas de normalidad multivariante, según la prueba de Mahalanobis (McLachlan, 1999), quedando una muestra final de 145 cuestionarios.

\section{Procesamiento de datos}

El procesamiento estadístico se realizó con SPSS 24 y AMOS, con la técnica multivariante de modelación de ecuaciones estructurales (SEM), porque analiza múltiples relaciones entre variables. Dicha técnica implica la estimación del modelo de medida y estructural. El primero comprueba la idoneidad de los ítems utilizados para evaluar cada constructo (colaboración externa vertical y horizontal y su relación con el desempeño financiero). El segundo comprueba las relaciones entre las variables latentes creadas (Weston \& Gore, 2006), es decir, la colaboración externa vertical y horizontal.

\section{Sujetos}

Los sujetos analizados fueron 145 propietarios y directivos de las pymes (pequeñas $75 \%$ y medianas $25 \%$ ) de los sectores industrial (23.45\%), comercial (48.28\%) y servicios (28.27\%), en Tamaulipas, en la frontera norte de México. Los datos recopilados fueron de cuestionarios 
completos, enviados digitalmente a 250 empresas a mediados del 2019, seleccionadas bajo el criterio de contar con cinco años o más de actividad económica.

Esto porque hay consenso en que la evaluación de innovación requiere un periodo de actividad empresarial. Los rasgos de los propietarios y directivos fueron: $73 \%$ hombres y $27 \%$ mujeres; $48 \%$ propietarios y $52 \%$ gerentes; en cuanto a formación académica, $1 \%$ manifestó no tener formación; $8 \%$, nivel medio superior; $77 \%$, licenciatura y $14 \%$, especialidad o posgrado.

\section{RESULTADOS Y DISCUSIÓN}

\section{Modelo de medida}

Se recurrió al análisis factorial confirmatorio para revisar fiabilidad y validez de escalas. Primero, se analizaron las cargas factoriales estandarizadas (CFE) para cada ítem de los constructos de colaboración externa vertical (Cev), horizontal (Ceh) y desempeño financiero (D), siendo eliminados aquellos con cargas bajo el valor mínimo aceptable de 0.700 (Hair, Black, Babin, \& Anderson, 2014).

Del constructo de colaboración externa vertical se eliminaron tres ítems (Cev3, Cev4, Cev7), de la colaboración externa horizontal se excluyeron tres ítems (Ceh9, Ceh 12 y Cehl4), y del desempeño financiero se eliminaron cuatro ítems (D1, D2, D3 y D4). Enseguida, se valoró la fiabilidad de las escalas de medición a través del alfa de Cronbach $(\alpha)$ y el índice de fiabilidad compuesto (IFC), los cuales obtuvieron valores superiores al mínimo exigido de 0.700 (Nunnally, 1978). Posteriormente, se revisó la validez convergente que demuestra una adecuada correlación entre ítems que componen un constructo, medida con la varianza promedio extraída (AVE), con valores sobre el umbral aceptable de 0.50 (Fornell \& Larcker, 1981).

En síntesis, se perciben tres resultantes a destacar en la Tabla 1. Primero, el desempeño financiero está determinado por el retorno sobre los activos, el retorno sobre las ventas y el retorno sobre la inversión, aspectos más relevantes considerados en la actividad financiera (Akhisar et al., 2015; Kalkan et al., 2014; Sánchez et al., 2015).

Segundo, la colaboración externa vertical se realiza con proveedores y clientes potenciales y actuales, lo cual es coherente con otros resultados empíricos (Bueno \& Balestrin, 2012; Chatenier et al., 2010; Chesbrough et al., 2006), aunque sin destacar los usuarios actuales y potenciales de los productos o servicios.

Tercero, la colaboración externa horizontal se manifiesta mediante la interacción con instituciones de educación, gobierno y competidores (Cancino \& Cárdenas, 2018; Lee et al., 2010), en el codesarrollo de productos o servicios, la obtención de conocimiento e ideas del entorno, la adquisición de equipos, maquinaria, software e insumos, y el uso de redes de intercambio de experiencias con instituciones educativas, gobierno y competidores, en acuerdo con otros estudios (Feller et al., 2009; Lee et al., 2012). 
Tabla 1. Validación de las escalas de medida

\begin{tabular}{|c|c|c|c|c|c|}
\hline Variable & Indicador & CFE & $\alpha$ & IFC & AVE \\
\hline \multirow{3}{*}{$\begin{array}{l}\text { Desempeño } \\
\text { financiero }\end{array}$} & D5. Retorno sobre activos & 0.842 & \multirow{3}{*}{0.895} & \multirow{3}{*}{0.896} & \multirow{3}{*}{0.743} \\
\hline & D6. Retorno sobre ventas & 0.880 & & & \\
\hline & D7. Retorno sobre inversión & 0.863 & & & \\
\hline \multirow{4}{*}{$\begin{array}{l}\text { Colaboración } \\
\text { externa vertical }\end{array}$} & Cev1. Clientes potenciales & 0.913 & \multirow{4}{*}{0.861} & \multirow{4}{*}{0.869} & \multirow{4}{*}{0.626} \\
\hline & Cev2. Clientes actuales & 0.666 & & & \\
\hline & Cev5. Proveedores actuales & 0.818 & & & \\
\hline & Cev6. Proveedores potenciales & 0.748 & & & \\
\hline \multirow{4}{*}{$\begin{array}{l}\text { Colaboración } \\
\text { externa horizontal }\end{array}$} & $\begin{array}{l}\text { Ceh8. Codesarrollo de productos o servicios con } \\
\text { instituciones de educación, gobierno o rivales }\end{array}$ & 0.683 & \multirow{4}{*}{0.800} & \multirow{4}{*}{0.804} & \multirow{4}{*}{$0.50 s$} \\
\hline & $\begin{array}{l}\text { Ceh10. Obtención de conocimiento e ideas con } \\
\text { instituciones de educación, gobierno o rivales }\end{array}$ & 0.697 & & & \\
\hline & $\begin{array}{l}\text { Ceh11. Adquisición de equipo, maquinaria, software e } \\
\text { insumos con instituciones de educación, gobierno o rivales }\end{array}$ & 0.834 & & & \\
\hline & $\begin{array}{l}\text { Ceh13. Redes de intercambio de experiencias con } \\
\text { instituciones académicas, gobierno y competidores }\end{array}$ & 0.624 & & & \\
\hline
\end{tabular}

Fuente: Elaboración propia a partir de resultados de AMOS.

En cuanto a la validez discriminante, permite demostrar diferencia de un constructo del resto en el modelo, y se analizó con la comparación del AVE y las correlaciones al cuadrado de cada constructo, siendo el AVE superior a las correlaciones recomendadas (Fornell \& Larcker, 1981), observado en la Tabla 2.

Tabla 2. Validez discriminante

\begin{tabular}{l|c|c|c}
\hline Constructos & $\begin{array}{c}\text { Desempeño } \\
\text { financiero }\end{array}$ & $\begin{array}{c}\text { Colaboración } \\
\text { externa vertical }\end{array}$ & $\begin{array}{c}\text { Colaboración } \\
\text { externa horizontal }\end{array}$ \\
\hline Desempeño financiero & 0.743 & & \\
\hline Colaboración externa vertical & 0.052 & 0.626 & \\
\hline Colaboración externa horizontal & 0.166 & 0.271 & 0.509 \\
\hline
\end{tabular}

Fuente: Elaboración a partir del AMOS.

\section{Modelo estructural}

La Tabla 3 recoge los indicadores de bondad de ajuste adecuados a Hair et al., (2014), obteniendo un ajuste incremental confiable de $\mathrm{CFI}=0.964$, TLI $=0.951, \mathrm{NFI}=0.918$, mayores al 0.900 recomendado. Por otra parte, se logró un buen ajuste absoluto, con un RMSEA=0.070, inferior al 0.0800 recomendado (Browne \& Cudeck, 1992). Finalmente, el ajuste de parsimonia determinado por chi-cuadrado /gl fue adecuado con un valor de 1.700 , siendo un valor menor a 2.000 . 
Tabla 3. Ajuste del modelo

\begin{tabular}{l|c|c|c}
\hline Estadistico & Valor & Abreviatura & Criterio \\
\hline fjuste absoluto & & X2 & Significación >0,050 \\
\hline Chi-cuadrado & $69.713(0.003)$ & RMSEA & $<0,080$ \\
\hline Raiz del residuo cuadrático promedio de aprox. & 0.070 & & $>0,900$ \\
\hline Ajuste incremental & & CFI & $>0,900$ \\
\hline Índice de bondad de ajuste comparativo & 0.964 & TLI & $>0,900$ \\
\hline Índice de Tucker-Lewis & 0.951 & NFI & \\
\hline Índice de ajuste normalizado & 0.918 & & (CMIN/DF) \\
\hline Parsimonia & & 1.700 & Menor que 3.000 \\
\hline Razón Chi-cuadrado/gl & & & $>$ \\
\hline
\end{tabular}

Fuente: Elaboración a partir del AMOS.

Los datos de la Tabla 4 muestran que la colaboración externa vertical tiene un bajo efecto positivo y no significativo en el desempeño financiero de las pymes ( $\mathrm{Hla}$ ), con un valor $\mathrm{P}$ de 0.831 (las hipótesis se aceptan con valores de $\mathrm{P}<0.05$ ), por lo cual se rechaza la afirmación de que la colaboración externa vertical de la innovación abierta influye positiva y significativamente en el desempeño financiero de las pymes, siendo un hallazgo diferente a investigaciones previas (Hippel, 2005; Khanagha et al., 2016; Schweisfurth \& Raasch, 2015).

Del hallazgo anterior, esto podría obedecer a que la relación colaborativa con clientes y proveedores se limita a diálogos informales sobre las actividades básicas (tipos de productos o servicios, especificaciones, precios, volúmenes, etc.), y no a actividades formales y complejas que implican cambios en productos o servicios, insumos, cadenas de suministros, etc. Lo anterior pone de manifiesto que este tipo de colaboraciones no siempre son relevantes para las empresas, o que requieren una apropiada configuración, recursos humanos, y agentes externos calificados, como lo aseveran otros estudios empíricos (Huang, Krull, \& Ziedonis, 2020; Raposo, Ferreira, \& Fernandes, 2014).

En tanto que la colaboración externa horizontal tiene un efecto positivo y muy significativo en el desempeño financiero, con un valor $\mathrm{P}$ de 0.001 (Hlb). Por lo que se confirma la hipótesis de que la colaboración externa horizontal de la innovación abierta influye positiva y significativamente en el desempeño financiero de las pymes. Lo anterior concuerda con trabajos realizados por Afcha (2011), Cancino y Cárdenas (2018) y Wang et al. (2015), en los cuales se afirma que involucrarse para el codesarrollo de productos o servicios con instituciones de educación (Vrande et al., 2009), gobierno (Lee et al., 2012) y competidores (Parida et al., 2012) genera valor financiero en estas empresas.

Asimismo, se destaca también la importancia de obtener conocimientos y diversidad de ideas técnicas y científicas a través del involucramiento con instituciones de educación, gobierno y competidores, pues se reduce el tiempo para adquirir el conocimiento necesario para afrontar 
nuevos retos, y se accede al conocimiento revelado, acelerando las curvas de aprendizaje y garantizando un mejor aprovechamiento de las capacidades tecnológicas, los resultados de I+D, y el rendimiento financiero (Hagedoorn et al., 2006; Tether, 2003).

También sobresalen las actividades de involucramiento en adquisición de equipos, maquinaria, software e insumos, pues impactan considerablemente en los resultados financieros. Esta relación positiva podría obedecer a que adquirir maquinara y equipo, software e insumos, así como realizar proyectos fuera de la cadena de valor, recibe más interés empresarial por sus implicaciones directas en indicadores financieros; por ejemplo, la colaboración con rivales minimiza los riesgos en el desarrollo de proyectos y permite compartir recursos, mejorando tiempos, costos e indicadores financieros (Alvarez-Aros \& Bernal-Torres, 2017; Lee et al., 2010; Mazzola et al., 2016).

Además de lo anterior, un elemento final a considerar es la participación en redes de intercambio de experiencias con instituciones académicas, gobierno y competidores, pues permite mejorar la formalización de la comunicación con agentes externos y, a su vez, se establecen límites organizacionales que garantizan una colaboración orientada hacia logros conjuntos de I+D e innovación, impactando en un mejor desempeño financiero (Chesbrough, 2015; Muller \& Hutchins, 2012; Ollila \& Yström, 2015).

Tabla 4. Resultados estimados

\begin{tabular}{l|l|l|l|c|c|c|c}
\hline Hipótesis & \multicolumn{2}{|l|}{ Relación variables endógenas } & Estimados estandarizados & S.E. & C.R. & P value \\
\hline $\mathrm{H} 1 \mathrm{a}$ & Desemp_financiero & $<--$ & CEV & 0.022 & 0.102 & 0.214 & 0.831 \\
\hline $\mathrm{H} 1 \mathrm{~b}$ & Desemp_financiero & $<--$ & CEH & 0.452 & 0.139 & 3.241 & 0.001 \\
\hline
\end{tabular}

Fuente: Elaboración propia a partir del AMOS

Complementario a la Tabla 4, la Figura 3 muestra los resultados estandarizados del modelo estructural, de las relaciones entre los ítems de las colaboraciones externas verticales y horizontales de las pymes con diferentes agentes exógenos, y la relación de esas colaboraciones con el desempeño financiero. Se observa el coeficiente de determinación $\left(\mathrm{R}^{2}\right)$ del modelo con un valor de 0.17 , lo que representa que las colaboraciones externas explican un $17 \%$ el desempeño financiero. Dicho coeficiente es clasificado como débil de acuerdo con Chin (1998), al ser inferior a 0.19, por lo tanto, el valor predictivo del modelo es bajo y representa una limitación del trabajo.

Sin embargo, debe indicarse que el desempeño financiero es un constructo complejo que es explicado por distintas variables relativas a la operación de cada organización y al mismo entorno que rodea a las empresas, y que han sido abordadas en la literatura organizacional. Por lo que en esta investigación solo se intenta determinar la parte de la varianza del desempeño financiero explicada por la colaboración externa. Al respecto, Hair et al., (2014) señalan que en un modelo con solo dos variables independientes y con un tamaño de muestra inferior a 250, un coeficiente de determinación $\left(\mathrm{R}^{2}\right)$ de $13 \%$ se considera estadísticamente significativo. 
Figura 3. Modelo estructural estandarizado

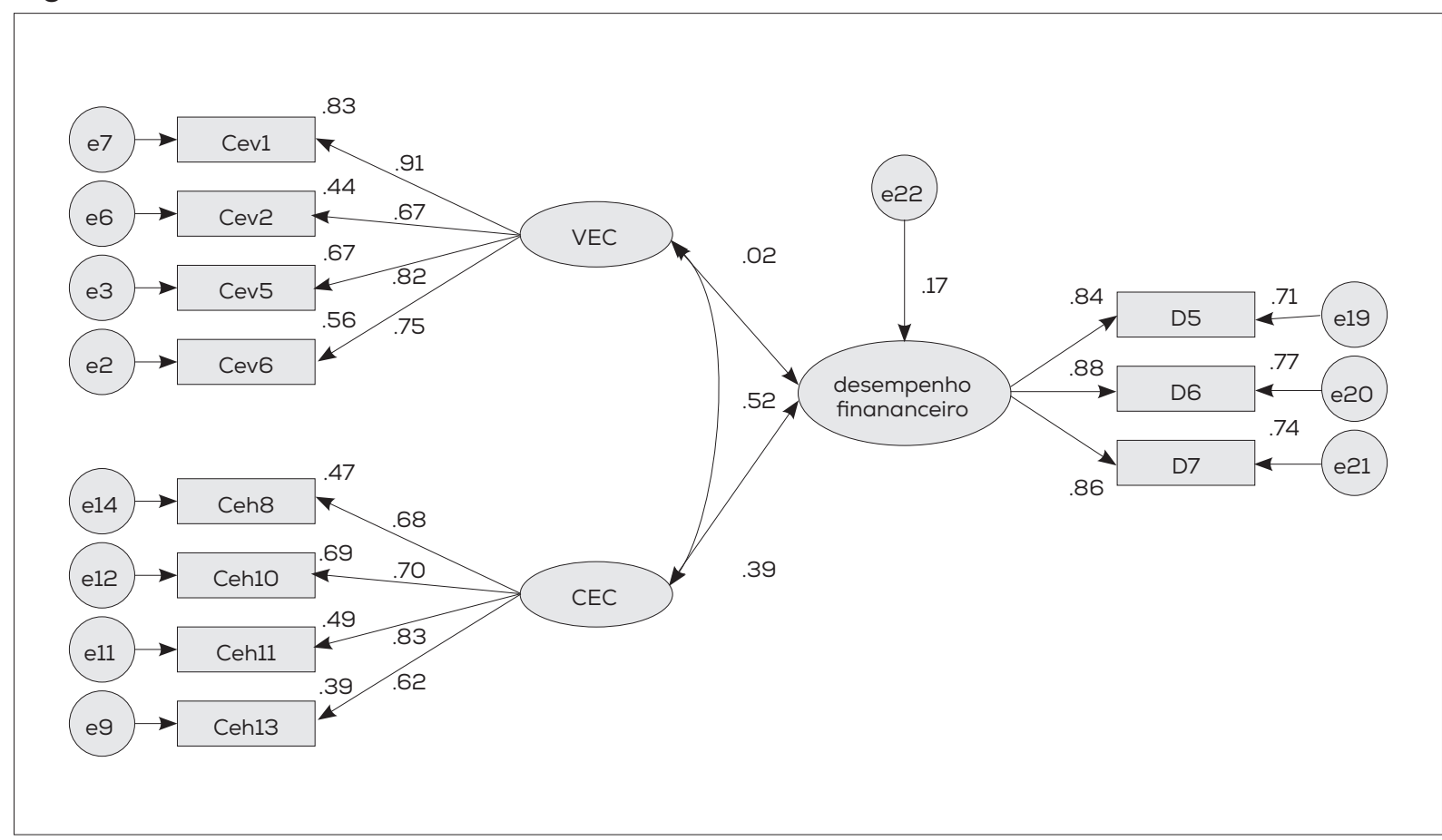

\section{CONCLUSIONES}

Este estudio contribuye a una mejor comprensión de las actividades de innovación abierta, concretamente la colaboración externa vertical y horizontal, y su relación con el desempeño financiero. A respecto de la Hla se encontró escaza relación positiva y no significativa entre la colaboración externa vertical con clientes, proveedores y usuarios, con los resultados financieros (Belderbos et al., 2010; Inauen \& Schenker-Wicki, 2011).

Esto probablemente se deriva de que la mayoría de la muestra de empresas en la frontera norte no son empresas industriales con perfil tecnológico, pues pertenecen sobre todo al sector comercial, donde se limitan a una configuración colaborativa de tratos básicos con usuarios, clientes y proveedores; y tratos comerciales más informales que no se destacan de la rutina operacional y se tornan sin relevancia para dicho sector (Huang, Krull, \& Ziedonis, 2020; Pipkin, 2018; Raposo et al., 2014).

Por otro lado, de la Hlb, como resultante novedoso, se confirmó una relación positiva y significativa desde el enfoque de la colaboración externa horizontal de la innovación abierta (instituciones de educación, gobierno y competidores) con relación a los indicadores del desempeño financiero. Esto manifiesta el aporte principal del estudio, por la importancia de actividades de involucramiento con estos agentes externos, desde la perspectiva de la teoría de la reducción de los costos transaccionales, como lo son las actividades de codesarrollo de productos y servicios, la obtención de conocimiento e ideas del entorno para mejorar la I+D y la innovación. 
También se destacan las actividades de adquisición de maquinaria, equipo, software e insumos, así como las redes de intercambio de experiencias hacia los resultados financieros de las pymes (Buganza \& Verganti, 2009; Lee et al., 2010, 2012).

Por consiguiente, esta investigación contribuye a la reflexión sobre la conciencia de los propietarios y directivos de las pymes en el trabajo colaborativo con agentes dentro y fuera del entorno en la innovación abierta. Esto representa una estrategia a potenciar para la configuración de la capacidad competitiva de negocios y en el rol del mercado limitado, donde suelen operar las pymes que parecieran no ser forzadas a realizar actividades colaborativas con actores externos. Dicha estrategia a potenciar mejoraría indudablemente el rol contextual donde las pymes realizan su actividad, sobre todo en una economía emergente, donde la cultura del trabajo colaborativo es aún incipiente.

Lo anterior, contrasta de los mercados amplios donde operan grandes empresas de economías desarrolladas, donde constantemente se exige trabajo colaborativo para innovar y potenciar la capacidad competitiva (García-Vidales et al., 2019; Stanisławskiy, 2020). Esto indica que el trabajo colaborativo en las pymes de las economías emergentes está determinado por la conciencia de los directivos, del rol del entorno, el tamaño del mercado y el contexto donde se desarrolla el mercado.

Estos resultados se suman a otras investigaciones que enfatizan la necesidad de investigar más al respecto, sobre todo en economías emergentes como México o economías no desarrolladas como sucede mayoritariamente con Latinoamérica (Scott \& Chaston, 2013). Por consiguiente, los hallazgos hasta ahora son poco concluyentes respecto al uso de colaboraciones externas horizontales y verticales para el mejoramiento del desempeño financiero en las pymes.

De lo anterior, es importante destacar que la búsqueda y adquisición de entradas, el uso de fuentes exógenas y la colaboración externa son estrategias de innovación abierta entrante, por lo cual estas actividades podrían estar al alcance de cualquier sector, ya sea comercial, industrial o de servicios. Esto porque las prácticas de la innovación abierta entrante están más orientadas a obtener recursos intangibles del ambiente exógeno (Alvarez-Aros \& Álvarez-Herrera, 2018).

También se aporta evidencia para la toma de decisiones empresarial, los directivos de entidades gubernamentales responsables del estímulo a estas empresas y a los académicos interesados en el tema, pues se requiere un esfuerzo en conjunto para seguir explorando las diferentes interacciones entre agentes externos, tomando en cuenta elementos como la amplitud y profundidad necesarias en dichas colaboraciones, que permitan un beneficio mutuo entre empresa y sociedad.

En cuanto a las limitaciones del estudio, es importante mencionar que los resultados obtenidos y las propuestas son válidas solo para la muestra analizada, y no se pueden generalizar. Además, este trabajo deja de lado actividades como el revelado de información, la propiedad intelectual, el licenciamiento, y los proyectos conjuntos, que son actividades propias de estrategias de innovación abierta saliente o mixta, si se complementan con la estrategia entrante, y representan una agenda futura como lo establecen otros trabajos (Alvarez-Aros \& Bernal-Torres, 2017).

Por último, otras futuras líneas de investigación son seguir estudiando el tema de las colaboraciones externas de la innovación abierta en ciudades fronterizas de economías 
emergentes o no desarrolladas de múltiples sectores empresariales, con estudios longitudinales y considerando además otros agentes externos como las instituciones bancarias, los centros de investigación privados, entre otros.

\section{REFERENCIAS}

Afcha, S. (2011). Innovaciones organizacionales y su efecto sobre el desempeño empresarial. Revista Venezolana de Gerencia, 16(56), 544-563. Recuperado de https://www.redalyc.org/articulo. oa?id=29020563003

Akhisar, İ., Tunay, K. B., \& Tunay, N. (2015). The effects of innovations on bank performance: The case of electronic banking services. Procedia-Social and Behavioral Sciences, 195(2015), 369-375. doi: 10.1016/j.sbspro.2015.06.336

Alvarez-Aros, E. L., \& Álvarez-Herrera, M. (2018). Estrategias y prácticas de la innovación abierta en el rendimiento empresarial: Una revisión y análisis bibliométrico. Investigación Administrativa, 47(121), 1-28. doi: 10.35426/IAv47n121.04

Alvarez-Aros, E. L., \& Bernal-Torres, C. A. (2017). Modelo de innovación abierta: Énfasis en el potencial humano. Información Tecnológica, 28(1), 65-76. doi: 10.4067/S0718-07642017000100007

Andersen, A. (1999). Diccionario de economía y negocios. Madrid: Editorial Espasa.

Armenteros, M. C., Elizondo, M. C., Medina, M., Ballesteros, L. L., \& Molina, V. (2012). Las prácticas de gestión de la innovación en las micro, pequeñas y medianas empresas: Resultados del estudio de campo en Piedras Negras Coahuila, México. Revista Internacional Administración y Finanzas, 4(5), 29-50. Recuperado de https://ssm.com/abstract=1954074

Aschhoff, B., \& Schmidt, T. (2008). Empirical evidence on the success of R\&D cooperation happy together? Review of Industrial Organization, 33(1), 41-62. doi: 10.1007/s1 1151-008-9179-7

Belderbos, R., Faems, D., Leten, B., \& Looy, B. Van. (2010). Technological activities and their impact on the financial performance of the firm: exploitation and exploration within and between firms. Journal of Product Innovation Management, 27(6), 869-882. doi:10.1111/j.1540-5885.2010.00757.x

Bernal-Torres, C. A., \& Frost-González, S. (2015). Innovación abierta en empresas colombianas: Reto a superar. Revista Venezolana de Gerencia, 20(70), 262-267. Recuperado de https://www.redalyc.org/ articulo.oa?id=29040281005

Browne, M. W., \& Cudeck, R. (1992). Alternative ways of assessing model fit. Sociological Methods \& Research, 21(2), 230-258. doi: 10.1177/0049124192021002005

Bueno, B., \& Balestrin, A. (2012). Collaborative innovation: An open approach in the development of new products. RAE-Revista de Administração de Empresas, 52(5), 517-530. doi: 10.1590/S003475902012000500004

Buganza, T., \& Verganti, R. (2009). Open innovation process to inbound knowledge: Collaboration with universities in four leading firms. European Journal of Innovation Management, 12(3), 306-325. doi:10.1108/14601060910974200 
Calderón, M. G. (2010). El valor estratégico de los acuerdos de colaboración para la adquisición de conocimiento en innovación abierta: Un análisis del sector de las TIC en España. Contaduría y Administración, (232), 4l-64. Recuperado de http://www.scielo.org.mx/scielo.php?script=sci_ arttext\&pid=S0186-10422010000300003\&lng=es\&tlng=es

Cancino, V., \& Cárdenas, J. (2018). Políticas y estrategias de vinculación con el medio en universidades regionales estatales de Colombia y Chile. Innovar, 28(68), 91-104. doi: 10.15446/innovar. v28n68.70474

Chatenier, E. D. Du, Verstegen, J. A., Biemans, H. J., Mulder, M., \& Omta, O. S. (2010). Identification of competencies for professionals in open innovation teams. R\&D Management, 40(3), 271-280. doi:10.1111/j.1467-9310.2010.00590.x

Chesbrough, H. W. (2003). Open innovation: The new imperative for creating and profiting from technology. Boston, USA: Harvard Business School Press.

Chesbrough, H. W. (2006). Open innovation: A new paradigm for understanding industrial innovation. In H. W. Chesbrough, W. Vanhaverbeke, \& J. West (Eds.), Open innovation: Researching a new paradigm (pp.1-12). New York, USA: Oxford University Press.

Chesbrough, H. W. (2015). Reinventar la empresa en la era digital. Innovación abierta. Innovar con éxito en el siglo XXI. OpenMind BBVA.

Chesbrough, H. W., Vanhaverbeke, W., \& West, J. (2006). Open innovation: Researching a new paradigm. New York, USA: Oxford University Press.

Chin, W. W. (1998). The partial least squares approach to structural equation modeling. In G. A. Marcoulides (Ed.), Modern methods for business research (pp. 295-333). Mahwah, USA: Lawrence Erlbaum.

Cohen, W. M., \& Levinthal, D. A. (1990). Absorptive capacity: A new perspective of learning and innovation. Administrative Science Quarterly, 35(1), 128-152. doi: 10.2307/2393553

Dahlander, L., \& Gann, D. (2010). How open is innovation? Research Policy, 39(6), 699-709. doi:10.1016/j.respol.2010.01.013

Das, T. K., \& Teng, B. S. (1998). Between trustand control: Developing confidence in partner cooperation in alliances. Academy of Management Review, 23(3), 491-512. doi:10.5465/AMR.1998.926623

Directorio Estadístico Nacional de Unidades Económicas, Instituto Nacional de Estadística y Geografía. (2019). Indicadores de ocupación y empleo. Recuperado de https://www.inegi.org.mx/contenidos/saladeprensa/boletines/2019/iooe/iooe2019_04.pdf

Enkel, E., Gassmann, O., \& Chesbrough, H. W. (2009). Open R\&D and open innovation: Exploring the phenomenon. R6D Management, 39(4), 311-316. doi:10.1111/j.1467-9310.2009.00570.x

Faems, D., Visser, M. De, Andries, P., \& Looy, B. Van. (2010). Technology alliance portfolios and financial performance: Value enhancing and cost increasing effects of open innovation. Journal of Product Innovation Management, 27(6), 785-796. doi:10.1111/j.1540-5885.2010.00752.x

Feller, J., Finnegan, P., Hayes, J., \& O’Reilly, P. (2009). Institutionalising information asymmetry: Governance structures for open innovation. Information Technology \& People, 22(4), 297-316. doi:10.1108/09593840911002423 
Fornell, C. \& Larcker, D. F. (1981). Evaluating structural equation models with unobservable variables and measurement error. Journal of Marketing Research, 18(1), 39-50. doi:10.2307/3151312

Fred, D. (2017). Conceptos de administración estratégica. Boletín Científico de las Ciencias Económico Administrativas del ICEA, 5(9)1-370. doi: 10.29057/icea.v4i8.217

Fry, C., Mention, A. L., Temel, S., \& Torkkeli, M. (2016). Exploring time lag effects of open innovation practices on performance during economic turmoil. International Journal of Business Innovation and Research, 10(2-3), 184-208. doi:10.1504/IJBIR.2016.074825

García-Vidales, M. Y., Maldonado-Guzmán, G., \& Pinzón-Castro, S. Y. (2019). The influence of open innovation practices on business performance in Mexican family and non-family SMEs. Estudios Gerenciales, 35(153), 370-378. doi: 10.18046/j.estger.2019.153.3202

Gassmann, O., Sandmeier, P., \& Wecht, C. H. (2006). Extreme customer innovation in the front-end: Learning from a new software paradigm. International Journal of Technology Management, 33(1), 46-66. doi:10.1504/IJTM.2006.008191

George, G., Zahra, S. A., \& Wood, D. R. (2002). The effects of business-university alliances on innovative output and financial performance: A study of publicly traded biotechnology companies. Journal of Business Venturing, 17, 577-609. doi:10.1016/S0883-9026(01)00069-6

Greco, M., Grimaldi, M., \& Cricelli, L. (2015). Open innovation actions and innovation performance: A literature review of European empirical evidences. European Journal of Innovation Management, 18(2), 150-171. doi:10.1108/EJIM-07-2013-0074

Hagedoorn, J., Roijakkers, N., \& Kranenburg, H. (2006). Inter-Firm R\&D networks: The importance of strategic network capabilities for high-tech partnership formation. British Journal of Management, 17(1), 39-53. doi: 10.1111/j.1467-8551.2005.00474.x

Hair, J. F., Jr., Black, W. C., Babin, B. J., \& Anderson, R. E. (2014). Multivariate data analysis (7ª ed.). Harlow, UK: Ed. Pearson-Prentice Hall.

Henkel, J. (2006). Selective revealing in open innovation processes: The case of embedded Linux. Research Policy, 35(7), 953-969. doi:10.1016/j.respol.2006.04.010

Hienerth, C. (2006). The commercialization of user innovations: The development of the rodeo kayak industry. RGD Management, 36(3), 273-294. doi: 10.1111/j.1467-9310.2006.00430.x

Hippel, E. Von. (2005). Democratizing innovation. Cambridge, USA: MIT Press.

Huang, J., Krull, L., \& Ziedonis, R. (2020). R\&D investments and tax incentives: The role of intra-firm cross-border collaboration. Contemporary Accounting Research. 37(4), 2523-2557. doi:10.1111/19113846.12588

Hung, K. P., \& Chiang, Y. K. (2010). Open innovation proclivity, entrepreneurial orientation, and perceived firm performance. International Journal of Technology Management, 52(3), 257-274. doi: 10.1504/IJTM.2010.035976

Hung, K. P., \& Chou, C. (2013). The impact of open innovation on firm performance: The moderating effects of internal R\&D and environmental turbulence. Technovation, 33(10), 368-380. doi: 10.1016/j. technovation.2013.06.006 
Hwang, J., \& Lee, Y. (2010). External knowledge search, innovative performance and productivity in the Korean ICT sector. Telecommunications Policy, 34(10), 562-571. doi: 10.1016/j.telpol.2010.04.004

Inauen, M., \& Schenker-Wicki, A. (2011). Fostering radical innovations with open innovation. European Journal of Innovation Management, 15(2), 212-231. doi: 10.1108/14601061211220986

Instituto Nacional de Estadística y Geografía. (2016). Producto interno bruto. Recuperado de www. inegi.org.mx

Kalkan, A., Bozkurt, Ö. Ç., \& Arman, M. (2014). The impacts of intellectual capital, innovation and organizational strategy on firm performance. Procedia - Social and Behavioral Sciences, 150, 700707. doi:10.1016/j.sbspro.2014.09.025

Khanagha, S., Volberda, H., \& Oshri, I. (2016). Customer co-creation and exploration of emerging technologies: The mediating role of managerial attention and initiatives. Long Range Planning, 50(2), 221-242. doi:10.1016/j.lrp.2015.12.019

Krause, W., \& Schuttle, C. S. L. (2015). A perspective on open innovation in small and medium sized enterprises in South Africa, and design requirements for an open innovation approach. South African Journal of Industrial Engineering, 26(1), 163-178. Recuperado de http://www.scielo.org.za/ scielo.php?script=sci_arttext\&pid=S2224-78902015000100013\&lng=en\&tlng=es

Laursen, K., \& Salter, A. (2006). Open for innovation: The role of openness in explaining innovation performance among U.K. manufacturing firms. Strategic Management Journal, 27(2), 131-150. doi: $10.1002 / \mathrm{smj} .507$

Lee, S., Park, G., Yoon, B., \& Park, J. (2010). Open innovation in SMEs: An intermediated network model. Research Policy, 39(2), 290-300. doi: 10.1016/j.respol.2009.12.009

Lee, S. M., Hwang, T., \& Choi, D. (2012). Open innovation in the public sector of leading countries. Management Decision, 50(1), 147-162. doi: 10.1108/00251741211194921

Maldonado-Guzmán, G., Madrid-Guijarro, A., Martínez-Serna, M. D. C., \& Aguilera-Enríquez, L. (2009). Los efectos de la innovación en el rendimiento de las mipymes de Aguascalientes: Una evidencia empírica. Revista de Economía, 1(1), 49-69. doi: 10.33937/reveco.2009.8

Marín-Idárraga, D. A., \& Cuartas-Marín, J. C. (2019). Relación entre la innovación y el desempeño: Impacto de la intensidad competitiva y el slack organizacional. RAE-Revista de Administração de Empresas, 59(2), 95-107. doi: 10.1590/s0034-759020190203

Martínez, S. M., Charterina, J., \& Araujo, A. (2010). Un modelo causal de competitividad empresarial planteado desde la VBR: Capacidades directivas, de innovación, marketing y calidad. Investigaciones Europeas de Dirección y Economía de la Empresa, 16(2), 1135-2523. doi: 10.1016/S11352523(12)60117-8

Martínez-Senra, A. I., Quintás, M. A., \& Caballero, G. (2014). La investigación básica en las empresas innovadoras españolas: Un análisis exploratorio. Innovar, 24(52), 79-88. doi: 10.15446/innovar. v24n 52.42524

Mazzola, E., Bruccoleri, M., \& Perrone, G. (2012). The effect of inbound, outbound and coupled innovation on performance. International Journal of Innovation Management, 16(6), 1240008. http:// doi.org/10.1142/S1363919612400087 
Mazzola, E., Bruccoleri, M., \& Perrone, G. (2016). Open innovation and firms performance: State of the art and empirical evidences from the bio-pharmaceutical industry. International Journal of Technology Management, 70(2-3), 109-134. doi: 10.1504/IJTM.2016.075152

McLachlan, G. J. (1999). Mahalanobis distance. Resonance, 4(6), 20-26. doi: 10.1007/BF02834632

Mention, A. L., \& Asikainen, A. L. (2012). Innovation \& productivity: Investigating effects of openness in services. International Journal of Innovation Management, 16(3), 27-53. doi: 10.1142/ S136391961240004X

Muller, A., \& Hutchins, N. (2012). Open innovation helps Whirlpool Corporation discover new market opportunities. Strategy \& Leadership, 40(4), 36-42. doi:10.1 108/10878571211242939

Nunnally, J. C. (1978), Psychometric theory. New York, USA: McGraw-Hill.

Obea. (2009). Innovación abierta. Más allá de la innovación tradicional. Universidad de Mondragón, MBA de la Facultad de Ciencias Empresariales. Obea Research Group. Recuperado de http://obea. blogs.mondragon.edu

Ollila, S., \& Yström, A. (2015). “Authoring” open innovation: The managerial practices of an open innovation director. Research in Organizational Change and Development, 23, 253-291. doi:10.1108/ S0897-301620150000023006

Parida, V., Westerberg, M., \& Frishammar, J. (2012). Inbound open innovation activities in high- tech SMEs: The impact on innovation performance. Journal of Small Business Management, 50(2), 283309. doi:10.1111/j.1540-627X.2012.00354.x

Park, S. H., \& Lee, Y. G. (2011). Perspectives on technology transfer strategies of Korean companies in point of resource and capability based view. Journal of Technology Management 6 Innovation, 6(1), 161-184. doi:10.4067/S0718-27242011000100013

Pipkin, S. (2018). Managing regional impacts of trade liberalization: Informal practices and collaborative economic development on the US-Mexico Border. Economic Development Quarterly, 32(2), 146162. doi:10.1177/0891242418770004

Quintana-García, C., \& Benavides-Velasco, C. A. (2004). Cooperation, competition, and innovative capability: A panel data of European dedicated biotechnology firms. Technovation, 24(12), 927-938. doi:10.1016/S0166-4972(03)00060-9

Raposo, M. L., Ferreira, J. J., \& Fernandes, C. I. (2014). Local and cross-border SME cooperation: Effects on innovation and performance. Revista Europea de Dirección y Economía de la Empresa, 23(4), 157-165. doi:/10.1016/j.redee.2014.08.001

Rodríguez, C., Terán, A., \& Bucci, N. (2011). La innovación abierta como elemento de análisis en las pequeñas y medianas industrias: Caso sector metal mecánico. Revista de Administração e Inovação, 8(2), 5-28. doi:10.5773/rai.v8i2.595

Rogo, F., Cricelli, L., \& Grimaldi, M. (2014). Assessing the performance of open innovation practices: A case study of a community of innovation. Technology in Society, 38, 60-80. doi:10.1016/j. techsoc.2014.02.006 
Romijn, H., \& Albaladejo, M. (2002). Determinants of innovation capability in small electronics and software firms in southeast England. Research Policy, 31(7), 1053-1067. doi:10.1016/S00487333(01)00176-7

Sánchez, Y., Zerón, M., \& Mendoza, G. (2015). Análisis del comportamiento estratégico y el desempeño organizacional en las pyme del centro de Tamaulipas en México. Revista Dimensión Empresarial, 13(1), 41-55. doi:10.15665/rde.v13i1.337

Schroll, A., \& Mild, A. (2011). Open innovation modes and the role of internal R\&D: An empirical study on open innovation adoption in Europe. European Journal of Innovation Management, 14(4), 475-495. doi:10.1108/14601061111174925

Schweisfurth, T. G., \& Raasch, C. (2015). Embedded lead users: The benefits of employing users for corporate innovation. Research Policy, 44(1), 168-180. doi:10.1016/j.respol.2014.09.007

Scott, G., \& Chaston, I. (2013). Open innovation in an emerging economy. Management Research Review, 36(10), 1024-1036. doi:10.1108/MRR-10-2011-0224

Sisodiya, S. R., Johnson, J. L., \& Grégoire, Y. (2013). Inbound open innovation for enhanced performance: Enablers and opportunities. Industrial Marketing Management, 42(5), 836-849. doi:10.1016/j.indmarman.2013.02.018

Souza, W. C. D., Junior, Torres, N., Júnior, \& Miyake, D. I. (2018). Servitization and organizational performance in the machinery and equipment sector. RAE-Revista de Administração de Empresas, 58(5) 475-493. doi:10.1590/S0034-759020180504

Stanisławski, R. (2020). Open innovation as a value chain for small and medium-sized enterprises: Determinants of the use of open innovation. Sustainability, 12(8), 3290. doi:10.3390/sul2083290

Tether, B. S. (2003). What is innovation? Approaches to distinguishing new products and processes from existing products and processes. 12 CRIC Working Centre for Research on Innovation and Competition. University of Manchester, USA.

Tsai, K. H. (2009). Collaborative networks and product innovation performance: Toward a contingency perspective. Research Policy, 38(5), 765-778. doi:10.1016/j.respol.2008.12.012

Ugarte-Cataldo, J. L. (2013). El concepto legal de empresa y el derecho laboral: Cómo salir del laberinto. Revista Chilena de Derecho Privado, (20), 185-213. doi:10.4067/S0718-80722013000100005

Vieira, A. L. (2014). Exploring data collection innovations by examining the effects of relationship marketing on performance in times of crisis. Innovar, 24(53), 75-82. doi:10.15446/innovar. v24n53.43911

Vrande, V. van de, Jong, J. P. de, Vanhaverbeke, W., \& Rochemont, M. de. (2009). Open innovation in SMEs: Trends, motives and management challenges. Technovation, 29(6), 423-437. doi:10.1016/j. technovation.2008.10.001

Wang, C. H., Chang, C. H., \& Shen, G. C. (2015). The effect of inbound open innovation on firm performance: Evidence from high-tech industry. Technological Forecasting and Social Change, 99, 222-230. doi:10.1016/j.techfore.2015.07.006 
Erick Leobardo Alvarez-Aros I César Augusto Bernal-Torres / Yesenia Sánchez Tovar

Weston, R., \& Gore, P. A., Jr. (2006). A brief guide to structural equation modeling. The Counseling Psychologist, 34, 719-751. doi:10.1177/0011000006286345

Yoon, B., Shin, J., \& Lee, S. (2016). Open innovation projects in SMEs as an engine for sustainable growth. Sustainability, 8(2), 146. doi:10.3390/su8020146

\section{CONTRIBUCIÓN DEL AUTOR}

Erick Leobardo Alvarez-Aros, César Augusto Bernal-Torres y Yesenia Sánchez Tovar trabajaron en la conceptualización y abordaje teórico-metodológico. La revisión teórica fue realizada por Erick Leobardo Alvarez-Aros y César Augusto Bernal-Torres. Erick Leobardo Alvarez-Aros coordinó la recopilación de datos y el primer análisis metodológico. El segundo análisis de datos y metodológico lo retrabajo Yesenia Sánchez Tovar. Todos los autores trabajaron juntos en la redacción de resultados y conclusiones y la revisión final del manuscrito. 